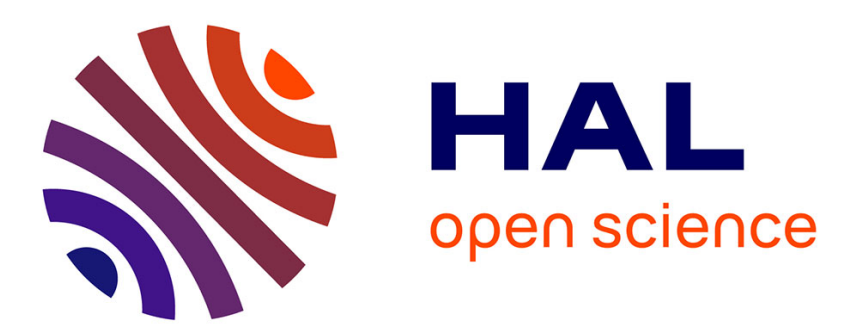

\title{
Depletion from a hard wall induced by aggregation and gelation.
}

\author{
Manuel Rottereau, Taco Nicolai, Jean-Christophe Gimel
}

\section{To cite this version:}

Manuel Rottereau, Taco Nicolai, Jean-Christophe Gimel. Depletion from a hard wall induced by aggregation and gelation.. European Physical Journal E: Soft matter and biological physics, 2005, 18, pp.37-40. 10.1140/epje/i2005-10028-4 . hal-00015710

\section{HAL Id: hal-00015710 https://hal.science/hal-00015710}

Submitted on 12 Dec 2005

HAL is a multi-disciplinary open access archive for the deposit and dissemination of scientific research documents, whether they are published or not. The documents may come from teaching and research institutions in France or abroad, or from public or private research centers.
L'archive ouverte pluridisciplinaire HAL, est destinée au dépôt et à la diffusion de documents scientifiques de niveau recherche, publiés ou non, émanant des établissements d'enseignement et de recherche français ou étrangers, des laboratoires publics ou privés. 


\title{
Depletion from a Hard Wall Induced by Aggregation and Gelation
}

\author{
Manuel Rottereau, Taco Nicolai, ${ }^{*}$ and Jean-Christophe Gimel \\ Polymères Collö̈des Interfaces, CNRS UMR6120, \\ Université du Maine, F-72085 Le Mans cedex 9, France
}

(Dated: December 12, 2005)

\begin{abstract}
Diffusion limited cluster aggregation and gelation of hard spheres is simulated using off-lattice Monte Carlo simulations. A comparison is made of the wall-particle correlation function with the particle-particle correlation function over a range of volume fractions, both for the initial system of randomly distributed spheres and for the final gel state. For randomly distributed spheres the correlation functions are compared with theoretical results using the Ornstein-Zernike equation and the Percus-Yevick closure. At high volume fractions $(\phi>40 \%)$ gelation has little influence on the correlation function, but for $\phi<10 \%$ it is a universal function of the distance normalized by correlation length $(\xi)$ of the bulk. The width of the depletion layer is about $0.5 \xi$. The concentration increases as a power law from the wall up to $r \approx \xi$, where it reaches a weak maximum before decreasing to the bulk value.
\end{abstract}

PACS numbers: 82.70.Dd,82.70.Gg

\section{INTRODUCTION}

Particles are depleted from the surface of a hard wall by excluded volume interaction. Depletion is an important phenomenon as it is the origin of flocculation and phase separation in a variety of systems [1] and can lead to slippage in shear experiments [2]. Depletion from a hard wall leads to a concentration profile: $C(r)=C \cdot g_{w}(r)$, where $g_{w}(r)$ is the wall-particle correlation function and $C$ is the number concentration in the bulk. In the case of hard spheres, $g_{w}(r)$ has been calculated by the same

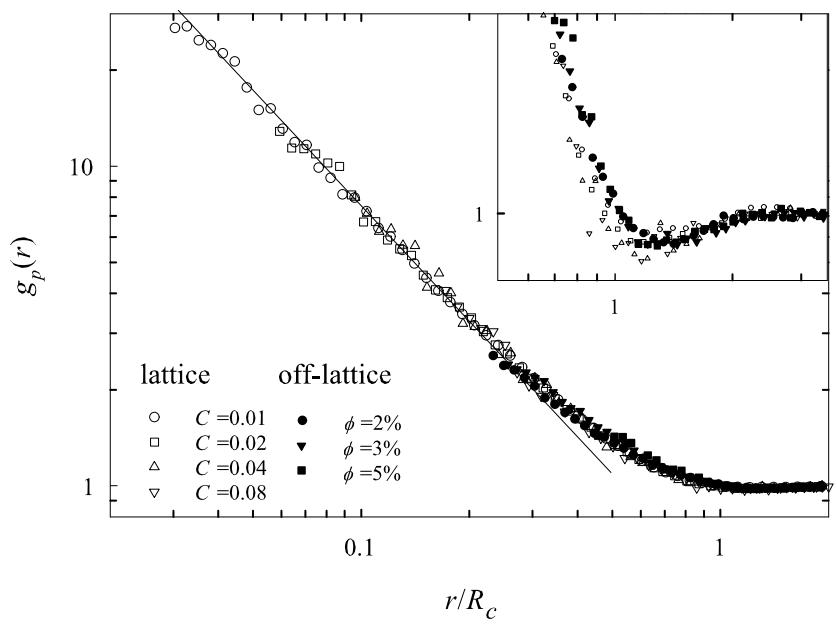

FIG. 1: Particle-particle correlation function for gels formed by DLCA at different volume fractions [13]. The straight line has slope -1.2 . The inset shows a blow-up of the minimum close to $r / R_{c}=1$

*Electronic address: Taco.Nicolai@univ-lemans.fr approach that was used to calculate the particle-particle correlation function $\left(g_{p}(r)\right)$, i.e. utilizing the OrnsteinZernike equation with the Percus-Yevick closure relation [3]. It was found that $g_{w}(r)$ resembles $g_{p}(r)$, except that the amplitude of the oscillations is lower for $g_{p}(r)$. An alternative approach is to calculate $g_{w}(r)$ within the framework of density functional theory [4].

Aggregation of the spheres has a profound influence on the structure of the system and one expects that it also influences the depletion from a hard wall. Aggregation of particles is a common phenomenon that leads to the formation of gels of widely different systems such as spherical [5] and disk-like [6] mineral particles, globular proteins [7] and emulsions [8]. Perhaps the simplest realistic model of aggregation is diffusion limited cluster aggregation (DLCA). In this model particles and clusters have Brownian motion and stick irreversibly every time they collide. Computer simulations and experiments [9] have shown that self similar aggregates are formed as long as they are dilute with a fractal dimension $d_{f}=1.8$ that relates their size $(R)$ to their mass $(m): m \propto R^{d_{f}}$.

However, when the clusters grow their cumulated volume fraction increases: $V_{\text {cum }} \propto R^{3-d_{f}}$, so that the average distance between the clusters decreases [10]. At some value of $R$ the clusters occupy the whole volume $\left(V_{\text {cum }} \approx 1\right)$ and interpenetrate. For strongly interpenetrated clusters the aggregation process that leads to gelation is well described by the static percolation model. Percolating clusters have a fractal dimension $d_{f}=2.5$ and are highly polydisperse [11]. After the gel point the clusters progressively collide with the system spanning gel until finally all particles are bound together.

Recently, DLCA has been studied in great detail using off-lattice Monte-Carlo simulations [12, 13]. It was found that the (broad) transition from clusters with $d_{f}=1.8$ to those with $d_{f}=2.5$ occurs at a characteristic radius of gyration $\left(R_{c}\right)$ that scales as expected with the particle volume fraction $(\phi=C \cdot \pi / 6): R_{c} \propto \phi^{1 /\left(d_{f}-3\right)}$, with $d_{f}=1.8$. The diameter of the spheres has been set to 

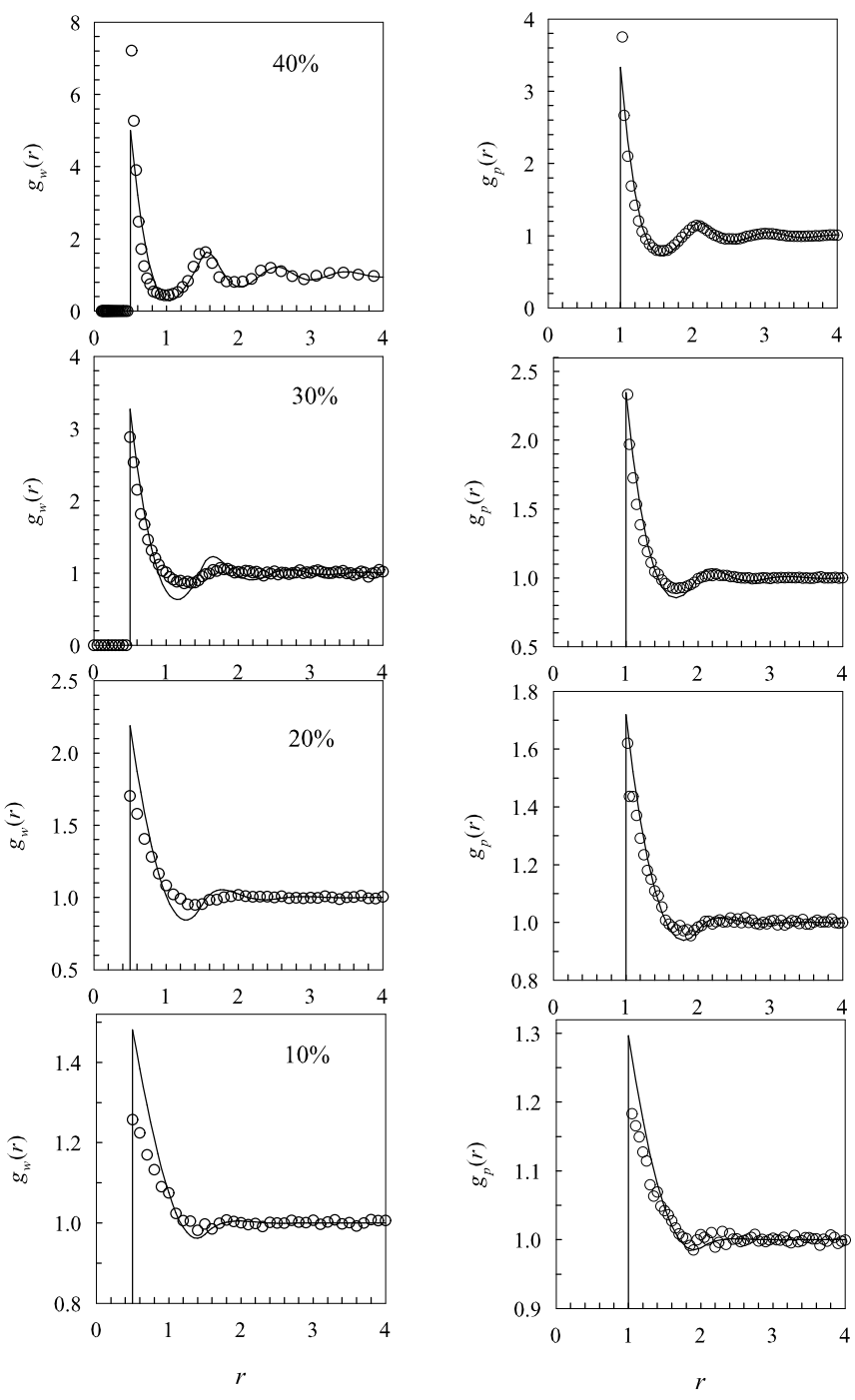

FIG. 2: Wall-particle (left) or particle-particle correlation functions (right) for randomly distributed hard spheres at different volume fractions. Notice the different scales of the y-axis. The solid lines represent Percus-Yevick predictions.

unity. $g_{p}(r)$ of the gels decreases following a power law for $r>3$ with slope $d_{f}-3$ showing the fractal structure at length scales below $R_{c}$. At $r \approx R_{c}, g_{p}(r)$ has a weak minimum before it reaches unity. A universal behaviour is found for $r>3$ if $g_{p}(r)$ is plotted as a function of $r / R_{c}$ both for lattice and off-lattice simulations, see Fig. 1. This universal behaviour can only be observed if $R_{c}$ is large, i.e. at low volume fractions. The structure at very small distances $(r<3)$ is determined by the detailed way in which the particles connect.

During the aggregation process the characteristic length scale, correlation length $(\xi)$, over which the system is fractal is initially proportional to the z-average radius of the aggregates, but stabilizes at a value close to $R_{c}$ when the aggregates interpenetrate and the gel is
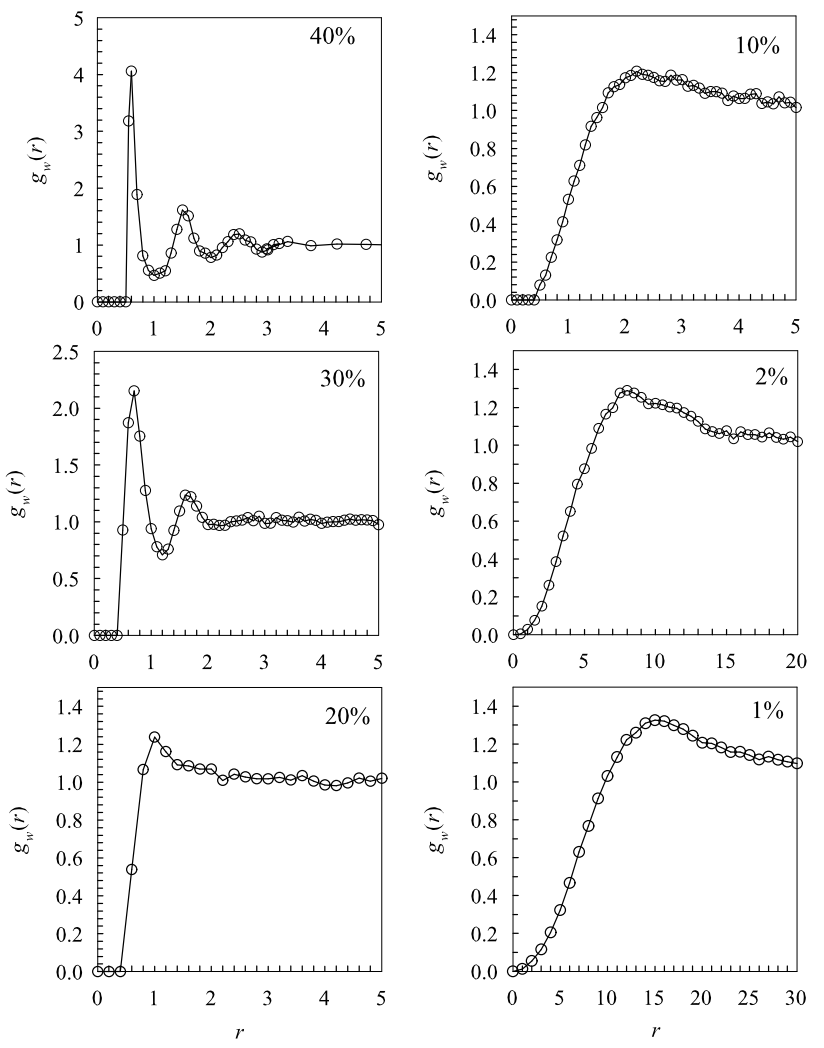

FIG. 3: Wall-particle correlation function for gels formed by DLCA at different volume fractions. Solid lines are guides to the eyes.

formed [13]. We may consider the system of interpenetrated aggregates or the gel as an ensemble of blobs with size $\xi$.

\section{RESULTS AND DISCUSSION}

Here we present a study of the influence of DLCA on the depletion layer at a hard wall using off-lattice MonteCarlo simulations. We will briefly describe the method we used, see [12] for more details. The simulations were done in a cubic box with size $L^{3}$. $N$ spheres with diameter unity are randomly positioned in the box leading to a number concentration $C=N / L^{3}$. A cluster is a collection of connected spheres. Unconnected spheres or clusters are randomly chosen and move in a random direction over a distance $s<<1$ with a probability inversely proportional to their diameter. If during a movement a sphere crosses an edge of the box, the movement is truncated at contact. If two spheres overlap, the movement is 

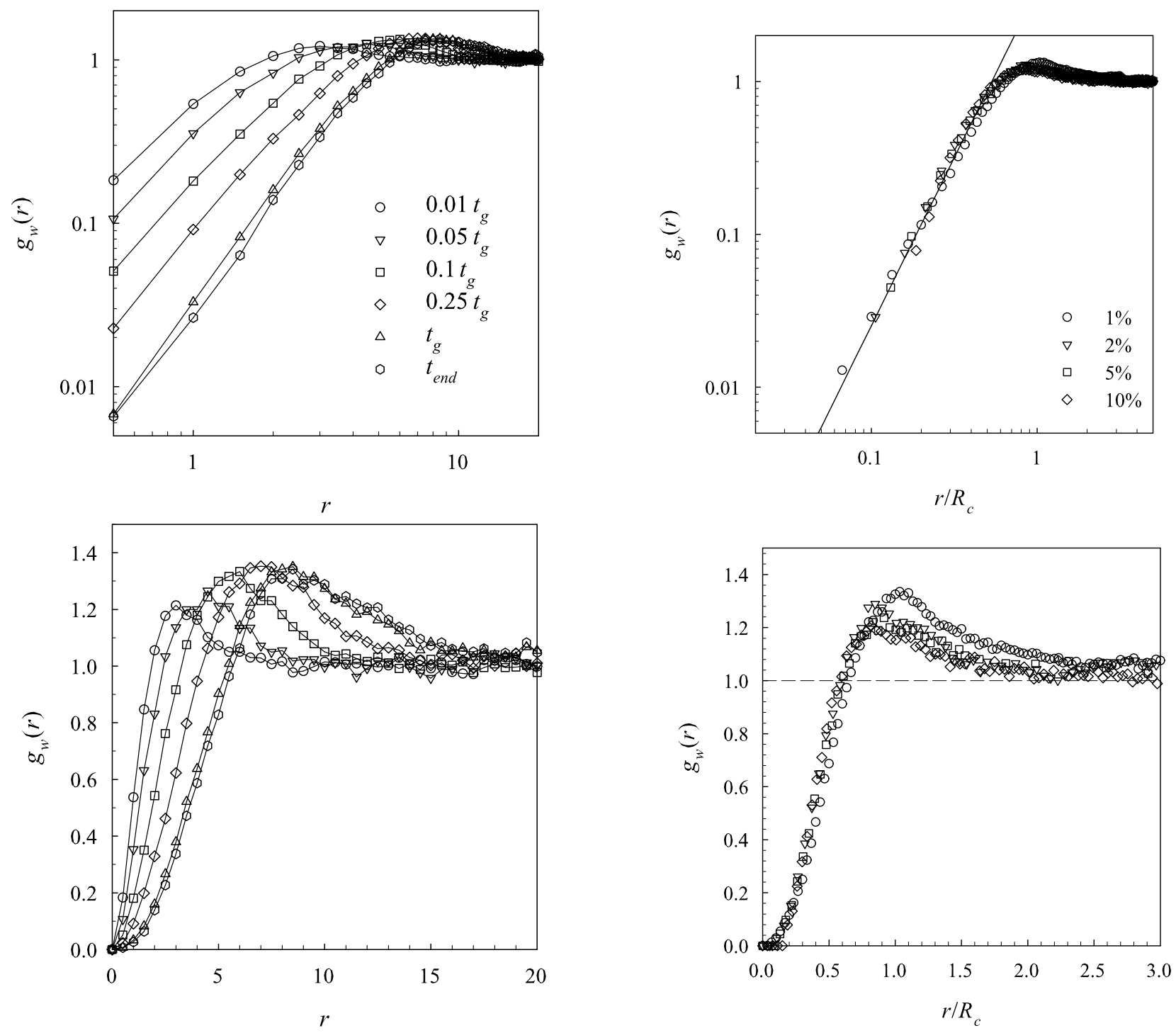

FIG. 4: Double logarithmic (top) and linear (bottom) representation of the wall-particle correlation function at $\phi=2 \%$ at different times during the aggregation. $t_{g}$ represents the gel time. Solid lines are guides to the eyes.

also truncated at contact and the spheres, that become part of a larger cluster, are connected together and now move collectively. This is repeated until the box contains a single cluster. We have used boxes of size $50 \leq L \leq 200$ and did not notice any finite size effects in the concentration range we studied $(1 \% \leq \phi \leq 40 \%)$. We have chosen $s<0.01$ and have observed an effect of the step size on $g_{p}(r)$ only for $1<r<1+s$ and on $g_{w}(r)$ only for $1 / 2<r<1 / 2+s$.

In Fig. 2 we compare $g_{w}(r)$ and $g_{p}(r)$ for the initial state of randomly distributed spheres at different volume fractions. Obviously, spheres are excluded for $r<0.5$ from the wall and for $r<1$ from another particle. For $r$ slightly larger there is an excess concentration of parti-

cles, which leads to a strongly damped oscillation of $g_{w}(r)$ and $g_{p}(r)$ with wavelength unity. The shape of $g_{p}(r)$ and $g_{w}(r)$ is similar, but the amplitude of the oscillations is somewhat larger for the latter. The extent of the depletion induced ordering decreases rapidly with decreasing volume fraction and is very small for $\phi<10 \%$. Solid lines represent the theoretical results using the OrnsteinZernike equation with the Percus-Yevick closure relation [3]. Small but significant differences are visible between the simulations and the theory both for $g_{p}(r)$ and $g_{w}(r)$.

In Fig. 3 we show $g_{w}(r)$ for the final gels formed by DLCA. As was discussed in [13], the structure is little influenced by aggregation at high concentrations, because the average distance between the sphere surfaces $(\Delta)$ de- 
creases strongly with increasing $\phi$ so that the aggregation process is terminated after the spheres have moved only over a small distance. In fact, the range of $r$ where modification can be expected is between $1 / 2$ and $1 / 2+\Delta$ for $g_{w}(r)$ and between 1 and $1+\Delta$ for $g_{p}(r)$.

Fig. 4 shows the development of the depletion layer at a low volume fraction ( $2 \%)$ during aggregation and gelation. The formation of the depletion layer mirrors that of the corresponding particle-particle correlation functions which can be found in Fig. 6 of [13]. Initially, the depletion layer broadens in proportion to the growth of the aggregates and its width is close to the radius of the aggregates. When the aggregates start to interpenetrate the width of the depletion layer is no longer proportional to the aggregate size, but to the correlation length of the system. After the gel point the correlation length of the system and therefore the depletion layer remains almost constant until the end of the process. Beyond the depletion layer a layer with excess particle concentration is formed. The maximum of $g_{w}(r)$ is situated at $r$ close to that of the minimum of the corresponding $g_{p}(r)$.

Fig. 3 shows that the depletion layer of the gels broadens with decreasing volume fraction. In view of the results obtained for $g_{p}(r)$, see Fig. 1, one might expect to observe universal behaviour if $g_{w}(r)$ is plotted as a function of $r / R_{c}$. Fig. 4 shows that a master curve is indeed obtained within the experimental error for $\phi<10 \%$. The same results are obtained with lattice simulations (not shown) because the details of the local structure are no longer important for $r>>1$. The width of the depletion layer is approximately $R_{c} / 2$, while the maximum is situated at $r \approx R_{c}$. In a double logarithmic representation one can clearly see that the depletion layer is characterized by a power law increase of $g_{w}(r)$ with exponent 2.2.

Early in the aggregation process the centre of mass of the aggregates is excluded from the wall up to a distance $r \approx R_{g}$. When $R_{g} \approx R_{c}$ the aggregates fill up the whole space and start to interpenetrate. Further aggregation does not involve movement over large distances and can essentially be described as a static percolation process. This is why the width of the depletion layer stagnates close to the gel point at a value of about $R_{c}$. Schematically we may consider the gel at contact with the wall as a layer of blobs with size $R_{c}$. If we assume that the fractal structure of the blobs in this surface layer is the same as in the bulk, then $g_{w}(r) \propto r^{3-d_{f}}$, which gives $g_{w}(r) \propto r^{1.2}$ for $d_{f}=1.8$. This is clearly not observed in the simulations, which implies that the structure of the gel close to the wall is different from the bulk.

The difference originates from the influence of the wall on the diffusion controlled aggregation process. Steric depletion induces a layer of excess concentration away from the wall. The layer with excess concentration acts as a sink for smaller clusters that are not sterically depleted from the wall. This is similar to the diffusion induced depletion layer around the clusters in the bulk. Therefore the depletion layer at the wall is both induced by diffusion and by excluded volume. The position of the excess concentration shifts to larger $r$ as the correlation length of the system grows until it stagnates at $R_{c}$. One might expect the maximum to be followed by a minimum caused by diffusion induced depletion. However, the minimum is very weak like in bulk.

\section{CONCLUSION}

We conclude that the width of the depletion layer of fractal aggregates and gels at a hard wall is close to the correlation length of the bulk systems. At low volume fractions the particle concentration increases from a very small value at the surface to the bulk concentration at $r \approx \xi$ following a power law with exponent 2.2. The depletion layer is followed by a layer with a maximum excess concentration of about $30 \%$. The depletion layer of gels formed by DLCA at different volume fractions is the same if the distance from the wall is scaled with $R_{c} \propto \phi^{d_{f}-3}$, using $\mathrm{df}=1.8$.

Acknowledgment. We thank D. Henderson for sending us a computer routine for the calculation of the theoretical correlation functions.
[1] S. Asakura and F. Oosawa, J. Polymer Sci. 33, 183 (1958); A. Vrij, Pure \& Appl. Chem. 48, 471 (1976).

[2] H. A. Barnes, J. Non-Newtonian Fluid Mech. 56, 221 (1995).

[3] D. Henderson, F. F. Abraham and J.A. Barker, Molec. Phys. 31, 1291 (1976).

[4] P.M. Köning, P. Bryk, K. Mecke and R. Roth, Europhys. Lett. 69, 832 (2005).

[5] M. Carpineti,F. Ferri, M. Giglio, E. Paganini and U. Perini Phys. Rev. A 42, 7347 (1990).

[6] T. Nicolai and S. Cocard, Eur. Phys. J. E 5, 221 (2001).

[7] C. G. de Kruif, M. A. M. Hoffmann, M. E. van Marle, P. J. J. M. van Mil, S. P. F. M. Roefs, M. Verheul and N. Zoon, Faraday Discuss 101, 185 (1995).
[8] S. H. Chen, J. Rouch, F. Sciortino and P. Tartaglia, J. Phys.: Condens. Matter 6, 10855 (1994).

[9] A. E. Gonzalez and G. Ramirez-Santiago, J. Colloid Interf. Sci. 182, 254 (1996) and references there in.

[10] J. C. Gimel, D. Durand and T. Nicolai, Phys. Rev. B 51, 11348 (1995).

[11] D. Stauffer and A. Aharony, Introduction to Percolation Theory, 2nd edn. (Taylor \& Francis, London, 1992).

[12] M. Rottereau, J. C. Gimel, T. Nicolai and D. Durand, Eur. Phys. J. E 15, 133 (2004).

[13] M. Rottereau, J. C. Gimel, T. Nicolai and D. Durand, Eur. Phys. J. E 15, 141 (2004). 